\title{
Hospital admission with pneumonia and subsequent persistent risk of chronic kidney disease: national cohort study
}

This article was published in the following Dove Press journal:

Clinical Epidemiology

\author{
Per-Ola Sundin' \\ Ruzan Udumyan' \\ Katja Fall ${ }^{1,2}$ \\ Scott Montgomery ${ }^{1,3,4}$ \\ 'Clinical Epidemiology and \\ Biostatistics, School of Medical \\ Sciences, Örebro University, Örebro, \\ Sweden; ${ }^{2}$ Medical Epidemiology and \\ Biostatistics, Karolinska Institutet, \\ Stockholm, Sweden; ${ }^{3}$ Clinical \\ Epidemiology Unit, Department \\ of Medicine, Karolinska University \\ Hospital Solna, Karolinska Institutet, \\ Stockholm, Sweden; ${ }^{4}$ Department \\ of Epidemiology and Public Health, \\ University College, London, UK
}

Background: Although acute onset kidney complications associated with severe infections including pneumonia are well characterized, little is known about possible subsequent delayed risk of chronic kidney disease (CKD).

Patients and methods: Associations between hospital admission with pneumonia in adulthood and raised risks of subsequent CKD were evaluated in a cohort of all male residents in Sweden born from 1952 to 1956 ( $\mathrm{n}=284,198)$ who attended mandatory military conscription examinations in late adolescence $(n=264,951)$ and were followed up through 2009. CKD and pneumonia were identified using Swedish national registers, and their associations were evaluated using Cox regression. Excluding the first year, the subsequent period was divided into $\leq 5,>5-\leq 15$, and $>15$ years after hospital admission with pneumonia. Follow-up ended on the date of first incident diagnosis of kidney disease, death, emigration, or December 31, 2009, whichever occurred first. Results: During a median follow-up of 36.7 (interquartile range 35.3-37.9) years from late adolescence, 5,822 men had an inpatient pneumonia diagnosis without contemporaneous kidney disease. Among exposed men, 136 (2.3\%) were later diagnosed with CKD compared with 2,749 $(1.2 \%)$ of the unexposed. The adjusted hazard ratio for CKD in the first year after the first episode of pneumonia was 14.55 (95\% confidence interval, 10.41-20.32), identifying early onset kidney complications and possibly pre-existing undiagnosed CKD. Starting follow-up 1 year after pneumonia to reduce the potential influence of surveillance bias and the risk of reverse causation, the adjusted hazard ratio for CKD in the first 5 years of follow-up was 5.20 (95\% confidence interval, 3.91-6.93) and then attenuated with increasing time.

Conclusion: Pneumonia among inpatients is associated with a persistently increased risk for subsequent $\mathrm{CKD}$, with the highest risk during the years immediately after pneumonia. Health care professionals should be aware of this period of heightened risk to facilitate early diagnosis and secondary preventive interventions.

Keywords: pneumonia, kidney disease, end-stage renal disease, inflammation, cohort study

\section{Introduction}

Acute onset kidney disease induced by infectious events has been recognized since the 19th century and is today extensively characterized and incorporated into our understanding of the pathophysiology of kidney disease. ${ }^{1}$ Despite increasing interest in the long-term effects of infections such as pneumonia, our knowledge in this area is incomplete for delayed risk of chronic kidney disease (CKD) ${ }^{2-6}$ Our review of the literature revealed only one study assessing the long-term risk of CKD following pneumonia, which investigated pneumococcal pneumonia and end-stage renal disease (ESRD) in Taiwan. ${ }^{7}$
Correspondence: Per-Ola Sundin Institutionen för Medicinska vetenskaper, Klinisk Epidemiologi och Biostatistik, Universitetssjukhuset Örebro, Campus USO, SE 70I 85 Orebro, Sweden $\mathrm{Tel}+4672 \quad 1436875$

Email perola.sundin@regionorebrolan.se 
Pneumonia affects $1.2 \%$ of the population in the northern hemisphere each year and constitutes the leading diagnosis requiring inpatient care in the US with approximately one million hospital admissions in the European Union annually ${ }^{8-10}$ Primary prevention is use of vaccines for the most important pathogen in community-acquired pneumonia, Pneumococcus pneumoniae. Pneumonia is associated with an increased risk of mortality, cardiovascular disease (CVD) and congestive heart failure for more than 10 years following infection. ${ }^{2-6,11}$ Temporal patterns with initial higher magnitude risks during the first 3-5 years have been identified for all-cause mortality and CVD while the temporal pattern for congestive heart failure risk is less consistent. ${ }^{2-5,11}$ The magnitude and persistence of the inflammatory response to pneumonia are associated with these adverse outcomes. ${ }^{6,12,13}$ Markers of raised inflammation levels have been associated with a higher risk of subsequent incident CKD, including our previous findings linking erythrocyte sedimentation rate in apparently healthy adolescent men with subsequent ESRD in middle age. ${ }^{14-17}$ Thus, inflammation and vascular disease, resulting from serious infections, may trigger not only acute post-infectious glomerulonephritis but also possibly initiate processes resulting in delayed kidney disease diagnosed several years later. ${ }^{1} \mathrm{CKD}$ is a public health issue of increasing importance throughout the world and is an important cause of death and loss of disability-adjusted life years. ${ }^{18}$ Therefore, if there is a period of heightened risk of disease onset during the years immediately following pneumonia, this may represent a target period for earlier diagnosis followed by secondary preventive interventions.

We examined whether an inpatient diagnosis of pneumonia in adult life is associated with a persistent risk of subsequent CKD in a representative birth cohort of 284,198 men followed into middle age, using routinely collected health data in national Swedish registers. To avoid surveillance bias and detecting acute pneumonia-related kidney complications, the main follow-up began 1 year after the pneumonia episode.

\section{Materials and methods Setting and participants}

A cohort of all male residents in Sweden born from 1952 to $1956(\mathrm{n}=284,198)$ who attended mandatory military conscription examinations in late adolescence $(n=264,951)$ was identified and followed up till December 31, 2009, using Swedish National Registers. ICD-8/9/10 codes, procedure codes and ATC codes for prescribed medications are presented in Table S1. All procedures involving patients and data were in accordance with the Helsinki Declaration of
1975 on ethical principles for medical research involving human subjects. ${ }^{19}$ The study was approved by the ethical review board of Uppsala, Sweden (numbers 2009/306 and 2014/324), which waived informed consent as the data were anonymized. Reporting of the study is in accordance with the STROBE statement. ${ }^{20}$

The Total Population Register provided dates of birth, death and emigration. The Swedish Military Conscription Register provided information on physical and psychological characteristics and diagnoses by late adolescence. The 1960 Population and Housing Census provided familial socioeconomic data during childhood. Routinely collected health data were extracted from the Swedish National Patient Register using ICD-8, ICD-9 and ICD-10 codes and procedure codes. Reporting to the National Patient Register is mandatory for all health care providers, which has information on inpatient diagnoses and procedures beginning in 1964, with complete national coverage since 1987 and with outpatient records since 2001..$^{21,22} \mathrm{In}$ addition to using the Patient Register, diagnoses of diabetes and hyperlipidemia were additionally also identified using the Swedish Prescribed Drug Register, which covers all prescribed medications collected by patients since July $1,2005 .{ }^{23}$

The cohort comprised ostensibly healthy adolescent men at the time of conscription examination with complete data for measures used in the analysis and without diagnosed kidney disease prior to pneumonia. Exclusions were made for data inconsistencies including incorrect personal identification number $(n=2,564)$; improbable measures at the conscription assessment ( $\mathrm{n}=225)$; no conscription assessment due to chronic illness, disability, lack of Swedish citizenship or incarceration $(\mathrm{n}=16,458)$; missing data $(\mathrm{n}=21,837)$; and conscription assessment outside ages $16-20$ years $(n=3,176)$. Men with diagnoses at conscription assessment consistent with kidney dysfunction, including trace or positive dipstick proteinuria; chronic inflammatory disease (vasculitis, rheumatoid arthritis and other collagenosis); and men with CVD or malformations of the urogenital or cardiovascular system at conscription assessment were excluded $(n=12,426)$. Finally, all men diagnosed with polycystic kidney disease at any time during follow-up were excluded $(n=148)$. A total of 227,364 men ( $80 \%$ of the birth cohort) remained in the analytical sample.

\section{Exposure}

After the conscription assessment, hospital discharge records were used to identify diagnoses of pneumonia without contemporaneous kidney disease in men without any previously recorded kidney disease. Diagnoses of pneumonia 
were considered as separate episodes if the discharge diagnoses were more than 30 days apart.

\section{Outcome}

The outcome CKD was based on the first occurrence of the following conditions in the National Patient Register: kidney failure, ESRD, glomerulonephritis, nephrotic syndrome, diabetic nephropathy, nephrosclerosis or hypertensive kidney disease. This wide definition was chosen due to the large proportion of CKD patients who receive a diagnosis without a kidney biopsy, which in our sample was $91.5 \%(2,639 / 2,885)$. ESRD was defined as long-term dialysis therapy, kidney transplantation, surgical procedures creating long-term access for dialysis or a diagnosis indicating stage $5 \mathrm{CKD}$.

\section{Covariates}

From the baseline conscription examination, established risk factors for CKD and measures previously associated with future ESRD in the same cohort were included as covariates. Erythrocyte sedimentation rate as determined by the Westergren method was adjusted for the erythrocyte volume fraction (microhematocrit method) in the analysis. ${ }^{24}$ Systolic blood pressure and diastolic blood pressure measured after rest in a recumbent position using a sphygmomanometer were categorized as optimal (systolic $<120$ $\mathrm{mmHg}$ and diastolic $<80 \mathrm{mmHg}$ ), normal (120-129/80-84 $\mathrm{mmHg}$ ), high-normal (130-139/85-89 mmHg), grade 1 hypertension (140-159/90-99 mmHg), grade 2 hypertension (160-179/100-109 $\mathrm{mmHg}$ ) and grade 3 hypertension (>180/>110 mmHg). ${ }^{25}$ Grades 2 and 3 hypertension were collapsed into a single category due to small numbers. Body mass index (BMI) categories were calculated from weight and height measured in light clothing without shoes. Physical working capacity was assessed using an electronically braked ergometer with the maximum load sustained for 6 minutes and summarized in a 0 - to 9 -point score. ${ }^{26} \mathrm{~A}$ cognitive function assessment covering linguistic understanding, spatial recognition, general knowledge and ability to follow mechanical instructions produced a 9-point score. ${ }^{27}$

Head of household's occupation and household crowding (quarters of the distribution of persons per habitable room ratio) in childhood from the 1960 census were included to account for socioeconomic conditions for the family of origin.

\section{Statistical analysis}

Descriptive statistics for exposed and unexposed men were compared using the two-sample $t$-test for continuous measures and Pearson's chi-squared test for categorical measures. ${ }^{28,29}$
Associations between hospital admission with pneumonia and subsequent CKD were evaluated using Cox regression with attained age as the underlying timescale. ${ }^{30}$ Follow-up began 1 year after the first episode of hospital admission with pneumonia, and the study population was followed up until date of first incident diagnosis of kidney disease, death, emigration or December 31, 2009, whichever occurred first. Due to evidence of non-proportional hazards, follow-up time was divided into categories ( $\leq 5,>5-\leq 15$ and $>15$ years). There was no evidence of violation of the proportional hazards assumption, tested using scaled Schoenfeld residuals, for these periods. ${ }^{31}$ For each period, model fit was tested using the added variable version of the Gronnesby and Borgan test. ${ }^{32}$ Adjusted models included all measures described in covariates section. All statistical analyses were conducted using STATA 14 (Stata Corp., College Station, TX, USA) or SPSS 22.0 (IBM Corp., Armonk, NY, USA).

\section{Sensitivity analyses}

The main analysis was repeated only considering pneumonia as the primary diagnosis at hospital discharge, and we also evaluated the effect of adjusting for repeated pneumonia episodes. Separate analyses excluded from the outcome acute kidney failure and diabetic nephropathy; and excluded from the study base were men ever diagnosed with sepsis, and men ever diagnosed with chronic obstructive pulmonary disease (COPD). Additionally, we restricted the outcome to ESRD, and collapsed the final two periods of follow-up time after pneumonia ( $>5-\leq 15$ and $>15$ years) due to low numbers. Additional adjustment was undertaken for CVD, diabetes and hyperlipidemia, modeled as time-dependent covariates.

To further tackle possible residual confounding by comorbidity and to reduce surveillance bias, the sample was restricted to include only men who had at least one hospital discharge diagnosis during adulthood. We applied incidence density sampling without replacement of unexposed to create nested matched cohorts. ${ }^{33}$ Each man with pneumonia (at risk of CKD) was matched with five unexposed men (at risk of CKD and pneumonia) by birth year, month and year of discharge (index date), censored at the time of first pneumonia diagnosis. ${ }^{34}$ In these nested cohorts, additional adjustments were included for adult diabetes and CVD recorded in the National Patient Register before or on the index date.

\section{Results}

The population is described in Table 1. During a median duration from study entry in late adolescence of 36.7 (interquartile range 35.3-37.9) years, 5,822 men had an 
Table I Characteristics of men with and without pneumonia

\begin{tabular}{|c|c|c|c|}
\hline Characteristic & Unexposed $(n=22 I, 542)$ & Exposed $(n=5,822)$ & $P$ \\
\hline CKD & $2,749(1.2 \%)$ & $136(2.3 \%)$ & $<0.001$ \\
\hline$C K D \geq I$ year after pneumonia & $\mathrm{n} / \mathrm{a}$ & $101(1.7 \%)$ & \\
\hline CKD $<$ I year after pneumonia & $\mathrm{n} / \mathrm{a}$ & $35(0.6 \%)$ & \\
\hline Years of follow-up before pneumonia & $\mathrm{n} / \mathrm{a}$ & $24.7(16.6-31.9)^{c}$ & \\
\hline \multicolumn{4}{|l|}{ Year of birth } \\
\hline 1952 & $43,469(19.6 \%)$ & I,274 (2I.9\%) & $<0.001$ \\
\hline 1953 & $43,086(19.4 \%)$ & I, $127(19.4 \%)$ & \\
\hline 1954 & $43,973(19.8 \%)$ & $\mathrm{I}, 135$ (19.5\%) & \\
\hline 1955 & $45,104(20.4 \%)$ & I, I 83 (20.3\%) & \\
\hline 1956 & $45,910(20.7 \%)$ & I, 103 (I8.9\%) & \\
\hline \multicolumn{4}{|l|}{ Age at conscription assessment (years) ${ }^{\mathrm{a}}$} \\
\hline 16 & $89(0.04 \%)$ & I $(<0.1 \%)$ & $<0.001$ \\
\hline 17 & $778(0.4 \%)$ & $17(0.3 \%)$ & \\
\hline 18 & $|09,32|(49.3 \%)$ & $2,566(44.1 \%)$ & \\
\hline 19 & $102,673(46.3 \%)$ & $2,917(50.1 \%)$ & \\
\hline 20 & $8,681(3.9 \%)$ & $321(5.5 \%)$ & \\
\hline \multicolumn{4}{|l|}{ Body mass index ${ }^{a}$} \\
\hline Underweight & 25,458 (11.5\%) & $772(13.3 \%)$ & $<0.001$ \\
\hline Normal & I79,574 (8I. I\%) & $4,518(77.6 \%)$ & \\
\hline Overweight & 14,373 (6.5\%) & 449 (7.7\%) & \\
\hline Obese & $2,137(1.0 \%)$ & $83(1.4 \%)$ & \\
\hline \multicolumn{4}{|l|}{ Hypertension $^{\mathrm{a}}$} \\
\hline Optimal & $39,484(17.8 \%)$ & $\mathrm{I}, 077$ (I8.5\%) & $<0.01$ \\
\hline Normal & $75,203(33.9 \%)$ & $2,067(35.5 \%)$ & \\
\hline High normal & $65,790(29.7 \%)$ & I,69I (29.0\%) & \\
\hline Grade I & $39,525(17.8 \%)$ & $952(16.4 \%)$ & \\
\hline Grade 2, 3 & $1,540(0.7 \%)$ & $35(0.6 \%)$ & \\
\hline \multicolumn{4}{|l|}{ Erythrocyte sedimentation rate ${ }^{\mathrm{a}}$} \\
\hline $0-1 \mathrm{~mm}$ & $52,462(23.7 \%)$ & I,387 (23.8\%) & 0.60 \\
\hline $2-6 \mathrm{~mm}$ & $146,275(66.0 \%)$ & $3,809(65.4 \%)$ & \\
\hline $7-10 \mathrm{~mm}$ & $14,603(6.6 \%)$ & $392(6.7 \%)$ & \\
\hline $\mathrm{II}-14 \mathrm{~mm}$ & $4,426(2.0 \%)$ & $119(2.0 \%)$ & \\
\hline$\geq 15 \mathrm{~mm}$ & $3,776(1.7 \%)$ & $115(2.0 \%)$ & \\
\hline Erythrocyte volume fraction ${ }^{a}$ & $46.4 I(2.4 I)$ & $46.48(2.45)$ & 0.04 \\
\hline Mean (SD) physical working capacity ${ }^{a}$ & $6.33(\mathrm{I} .8 \mathrm{I})$ & $6.05(1.83)$ & $<0.001$ \\
\hline Mean (SD) IQ score ${ }^{a}$ & $5.22(1.97)$ & $4.80(2.05)$ & $<0.00 \mathrm{I}$ \\
\hline \multicolumn{4}{|l|}{ Household crowding (quantiles) ${ }^{\mathrm{b}}$} \\
\hline I Lowest & $57,715(26.1 \%)$ & I,382 (23.7\%) & $<0.00 \mathrm{I}$ \\
\hline 2 & $68,616(31.0 \%)$ & I,77I (30.4\%) & \\
\hline 3 & $47,272(21.3 \%)$ & I,292 (22.2\%) & \\
\hline 4 Highest & $47,939(21.6 \%)$ & I,377 (23.7\%) & \\
\hline \multicolumn{4}{|l|}{ Head of household's occupation ${ }^{\mathrm{b}}$} \\
\hline Manual/agricultural work & $100,034(45.2 \%)$ & $2,751(47.3 \%)$ & $<0.00 \mathrm{I}$ \\
\hline Farm owners/managers & $22,308(10.1 \%)$ & $513(8.8 \%)$ & \\
\hline Office workers & $61,767(27.9 \%)$ & I,549 (26.6\%) & \\
\hline Business owners/managers & $24,025(10.8 \%)$ & $587(10.1 \%)$ & \\
\hline Others & $13,408(6.1 \%)$ & $422(7.2 \%)$ & \\
\hline
\end{tabular}

Notes: Values are numbers (with \%) unless stated otherwise. alndicates measures from conscription assessment. bIndicates measures in childhood. Median (interquartile range). Groups are compared using Pearson's chi-squared test and continuous measures using two-sample $t$-test.

Abbreviations: CKD, chronic kidney disease; n/a, not applicable.

inpatient pneumonia diagnosis without contemporaneous kidney disease. Among exposed men, 136 were diagnosed with CKD (19.34 per 10,000 person-years, 95\% confidence interval 16.35-22.88) compared with 2,749 of the unexposed men (3.47 per 10,000 person-years, $95 \%$ confidence interval 3.40-3.60), representing an increased relative risk of $88.3 \%$. No men were lost to follow-up within Sweden since the unique Swedish personal identification number allows 
full coverage in national registers. The pattern of censoring of exposed and unexposed men is included in Figure 1. The Gronnesby and Borgan test did not indicate inadequate model fit for any of the periods following exposure $(P>0.05)$. The unadjusted and adjusted hazard ratios for CKD in the excluded first year after pneumonia are 15.81 (11.32-22.07) and 14.55 (10.41-20.32), respectively, identifying early onset kidney complications and possibly pre-existing undiagnosed kidney disease. Before the end of follow-up, 19.9\% of the exposed men with CKD progressed to ESRD.

Starting follow-up 1 year after pneumonia to reduce surveillance bias and the risk of reverse causation, the adjusted hazard ratio for CKD diagnosed in the first 5 years of follow-up is 5.20 (3.91-6.93). Hazard ratios for CKD following pneumonia are presented in Table 2. There is an initial high magnitude risk during the first 5 years, which attenuates somewhat over time, but remains statistically significant more than 15 years after the pneumonia diagnosis. All covariates are statistically significantly associated with CKD in unadjusted analysis. The most notable associations with increased risk for $\mathrm{CKD}$ in the adjusted model are high BMI, low physical working capacity, low cognitive function score and greater household crowding (Table S2). Adjustment has no major influence on the association of pneumonia with CKD.

\section{Sensitivity analyses}

Results from the sensitivity analyses performed in the main cohort are included in Table 2. After additional adjustment for CVD, diabetes and hyperlipidemia as time-dependent covariates, the magnitudes of the hazard ratios for the main results were attenuated moderately but they remained statistically significant for all time periods between pneumonia and CKD; the results look more similar to those from the matched hospital cohort. The remaining sensitivity analyses do not indicate any notable change in the associations; the association of pneumonia with CKD is not explained by multiple pneumonia episodes. When restricting the outcome to ESRD, the unadjusted and adjusted hazard ratios during the first 5 years of follow-up were $6.22(3.42-11.32)$ and 5.72 (3.14-10.41), respectively, attenuating to $2.20(1.17-4.12)$ and 2.15 (1.15-4.03) in the remaining years of follow-up. The nested matched hospital cohorts comprised $99.9 \%$ $(5,814 / 5,822)$ of the exposed men, who could be matched successfully with five unexposed men. Median follow-up was 10.7 years (interquartile range 3.8-18.6). Characteristics of the nested matched cohorts (men were inpatients in both cohorts) are given in Table $\mathrm{S} 3$, and censoring patterns are detailed in Table S4. The unadjusted hazard ratio for CKD during the first 5 years of follow-up was 2.81 (1.97-4.02) and using a model adjusted for characteristics in childhood and adolescence, and CVD and diabetes in adulthood, the hazard ratio was 2.85 (1.84-4.41). Hazard ratios during the following 10 years of follow-up were $2.13(1.42-3.20)$ and 1.75 (1.09-2.82) for unadjusted and adjusted models, respectively.

\section{Discussion}

In this large national cohort study of men in Sweden, hospital admission with pneumonia was associated with a persistent increased risk for subsequent $\mathrm{CKD}$, particularly during the

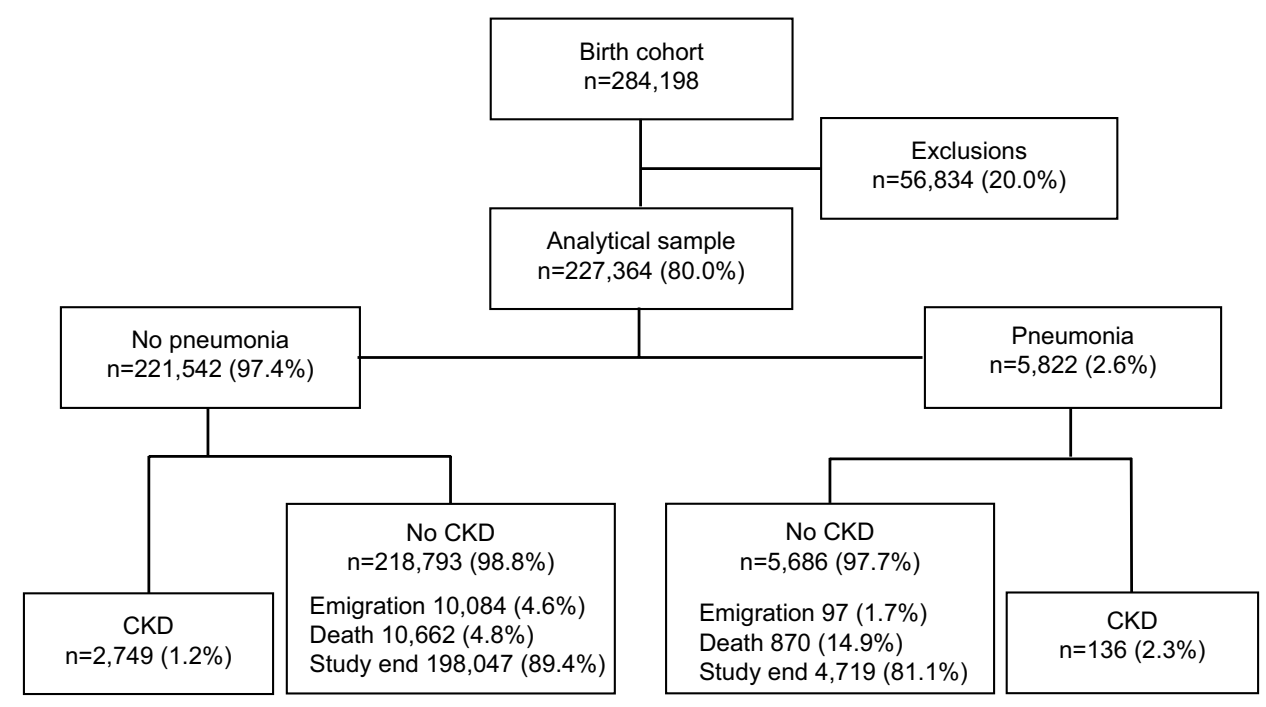

Figure I Study flow chart including censoring. Abbreviation: CKD, chronic kidney disease. 
Table 2 Associations of pneumonia with chronic kidney disease during time periods following hospital admission with pneumonia

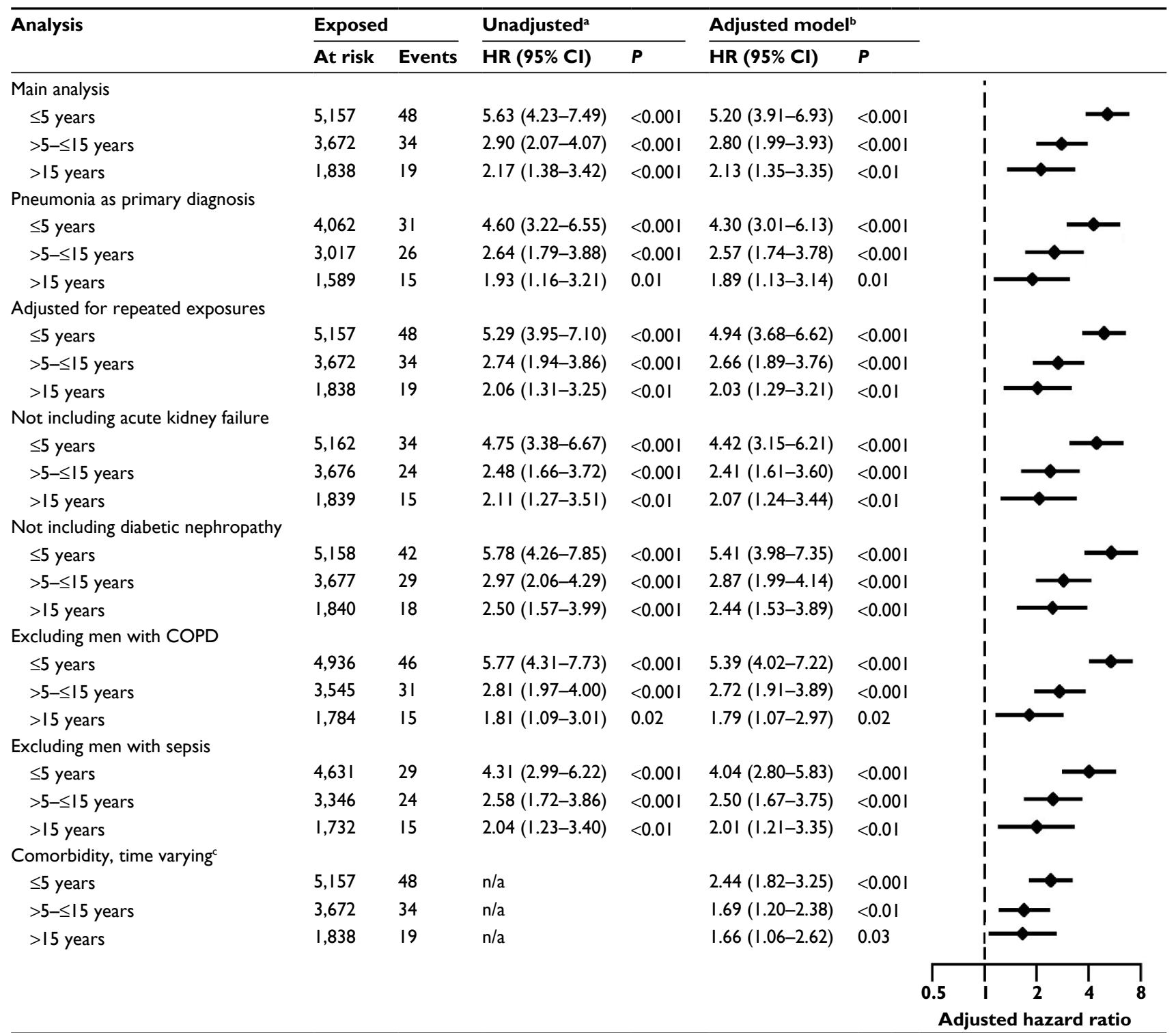

Notes: aFollow-up time starting I year after pneumonia. ${ }^{b}$ Adjusted for characteristics in adolescence (body mass index, blood pressure, physical working capacity, cognitive function, erythrocyte sedimentation rate, erythrocyte volume fraction) and childhood (household crowding and head of household's occupation). 'Additionally adjusted for cardiovascular disease, diabetes and hyperlipidemia as time varying covariates.

Abbreviations: $\mathrm{Cl}$, confidence interval; COPD, chronic obstructive pulmonary disease; HR, hazard ratio.

first 5 years of follow-up beginning 1 year after the infection. Although attenuated over time, the raised risk remained statistically significant for more than 15 years. The association was independent of measures in childhood and adolescence previously associated with ESRD in the same cohort. ${ }^{17}$ The association was robust, as indicated by the results of a number of sensitivity analyses addressing possible confounding by comorbidity, repeated pneumonia episodes, and sepsis or acute kidney failure. The results from an additional analysis of nested matched cohorts of men who had all been hospital inpatients indicate that neither contemporaneous diabetes or CVD at the time of the pneumonia episode nor surveillance or selection bias seem to explain the findings. Further analysis of the main cohort with additional adjustment for timing of diagnoses for diabetes, CVD and hyperlipidemia suggest that although these types of comorbidity are associated with a higher magnitude risk of CKD following pneumonia, a history of inpatient care with pneumonia identifies a statistically significant independent risk. The maximum age of men in this study was only 57 years, and so a larger proportion with pneumonia and CKD may be expected in an older population.

Our study has potential limitations. Given the limitations of the administrative dataset, the CKD outcome comprises a heterogeneous mix of diagnoses rather than information on 
estimated glomerular filtration rate and proteinuria, which would provide higher sensitivity for detection of CKD and allow grading of CKD stage. However, treating these diagnoses as a common entity rather than separate outcomes is reasonable, given the low number of kidney biopsies performed in clinical practice and diagnoses based on reduced kidney function without identifying the etiology. Sensitivity analysis excluding some diagnoses did not alter the findings notably. The binary nature of the CKD outcome limits evaluation of its clinical significance, although increasing severity of kidney disease would be recorded in the discharge summary to an increasing extent. The results were similar when the outcome was limited to ESRD. The potential underestimation of the incidence of CKD applies equally to exposed and unexposed men and would be expected to dilute rather than cause a spurious association. Non-detection of pre-existing $\mathrm{CKD}$ at the pneumonia episode may also introduce reverse causation. However, the increased susceptibility to infections associated with CKD is mainly seen in moderate and advanced disease, which should be recorded in the discharge summaries. ${ }^{35,36}$ Exclusion of CKD diagnoses in the first year after discharge will also tackle this problem, even though it produces a substantially lower magnitude association. Thus, our main findings are likely to underestimate the risk of CKD following pneumonia, but this is preferable to the spuriously inflated estimates that may occur if the first year after infection is included. Hospital discharge records do not separate acute kidney failure with complete remission from cases resulting in $\mathrm{CKD}$. In sensitivity analyses excluding acute kidney failure from our definition of CKD, no effect on the associations was evident. Pneumonia is associated with an increased short-term and long-term mortality and we did not evaluate competing risks. ${ }^{4,11,13}$ Although the competing event, death without diagnosed CKD, occurred in a substantially higher proportion of exposed men, it does not change our key finding of an increased risk of CKD following pneumonia for men still at risk.

Measures of proteinuria, BMI and blood pressure were only available from the initial conscription assessment and we did not have information on smoking. Our results were robust in sensitivity analysis excluding patients with COPD and signaling smoking, although of course not identifying all smokers. There is a possibility of confounding by comorbidity, but exclusion of events in the first year after pneumonia, sensitivity analysis with additional adjustment for CVD, diabetes and hyperlipidemia as time-dependent covariates and creation of matched hospital inpatient cohorts allowing adjustment for additional risk factors in adulthood make this less likely to be a major limitation. Comparing hospital cohorts may underestimate the magnitude of risk as the unexposed hospital patients may carry more risks for CKD than the general population. Whether or not hospital admission with pneumonia has a causal association with CKD, it serves to identify patients at increased risk of developing CKD. The study population comprised only men due to the use of military conscription assessment data. There are potential sex differences in the underlying pathophysiology of CKD, which may limit the generalizability of our results to women. ${ }^{37,38}$

Our study also has several major strengths, including characterization of the temporal pattern of the association between pneumonia and $\mathrm{CKD}$, the large general population based sample of men, long-term follow-up, the availability of initial clinical conscription assessment data before follow-up and the flexibility to create nested cohorts of men who were all admitted to hospital to further tackle potential surveillance and selection bias.

To our knowledge, only one previous study has evaluated the potential long-term risks for CKD following pneumonia in adults. Huang et al performed a population-based retrospective cohort study based on claims data in Taiwan's National Health Insurance Research Database to assess ESRD risk during 13 years of follow-up for patients admitted to hospital with pneumococcal pneumonia. ${ }^{7}$ The unexposed were not required to be inpatients. The overall adjusted hazard ratio for ESRD associated with pneumonia (1.14, 95\% confidence interval: 1.01-1.29) indicated a statistically significant slight increase in ESRD risk. ESRD risk in different time-periods following pneumonia was not evaluated. Taiwan has the highest incidence and prevalence of ESRD in the world, despite a prevalence of CKD comparable to other Asians and western countries and this has been suggested to indicate an underestimation of CKD prevalence in Taiwan. ${ }^{39}$ There are also differences in the pattern of CKD etiology between Asia and Europe, including a more important role for chronic infections (HIV, hepatitis), CKD from herbal medications and tropical infections with potential kidney sequelae. ${ }^{39,40}$ Our study undertaken in a European setting adds characterization of the temporal pattern of risk, evaluation of not only ESRD but CKD risk in general, a longer follow-up, addresses potential selection and surveillance bias in the nested matched hospital cohorts, and is not restricted to pneumococcal pneumonia.

There are several possible explanations for the notable increase in CKD risk associated with hospital admission with pneumonia. Individuals with immunodeficiency are at increased risk of pneumonia and there is also an association between immunodeficiency and autoimmunity, possibly 
causing CKD. ${ }^{1,41}$ However, more severe immunodeficiency is likely to be associated with repeated episodes of hospital admissions with pneumonia, and adjusting for repeated exposures had no major effect on the associations. Several markers of inflammation are associated with future incident CKD, and progression of established CKD and pneumonia has the potential to cause persistent low-grade systemic inflammation. Although the majority of inpatients with pneumonia recover clinically within 1 week, markers of systemic inflammation remain elevated in more than $50 \%$ of patients; the mean circulating C-reactive protein level 6 months after pneumonia has been reported to be elevated $(5 \mathrm{mg} / \mathrm{L}){ }^{12,42,43}$

Our results have implications for clinicians and policy makers. Increased risks for mortality, CVD, congestive heart failure and, as indicated by our study, CKD extend for at least a decade after an episode of pneumonia. ${ }^{2-6}$ Increased awareness of the initial period of high-magnitude CKD risk following pneumonia could facilitate early diagnosis and secondary preventive interventions. The expanding insights into the adverse consequences of pneumonia may necessitate a future re-evaluation of the costs and benefits of pneumococcal vaccination.

\section{Conclusion}

Hospital admission with pneumonia in adulthood indicates a future increased risk of CKD, most notably during the first 5 years, indicating a possible target period for early detection and secondary preventive interventions.

\section{Data sharing statement}

The ethical approval does not allow data sharing. The registry data can be applied for through official channels.

\section{Acknowledgments}

This study received support from the UK Economic and Social Research Council as grants to the International Centre for Life Course Studies (grants RES-596-28-0001 and ES/JO19119/1). The study sponsor had no role in the study design; collection, analysis, and interpretation of data; writing of the report; or decision to submit the article for publication.

\section{Author contributions}

POS and SM designed the study; RU, SM and KF acquired and prepared the data; POS analyzed the data; POS, SM, KF and RU interpreted the data; SM supervised the study. POS wrote the first draft of the manuscript to which all authors made significant subsequent contributions. All authors contributed toward data analysis, drafting and critically revising the paper, gave final approval of the version to be published, and agree to be accountable for all aspects of the work.

\section{Disclosure}

The authors report no conflicts of interest in this work.

\section{References}

1. Couser WG, Johnson RJ. The etiology of glomerulonephritis: roles of infection and autoimmunity. Kidney Int. 2014;86(5):905-914.

2. Corrales-Medina VF, Alvarez KN, Weissfeld LA, et al. Association between hospitalization for pneumonia and subsequent risk of cardiovascular disease. JAMA. 2015;313(3):264-274.

3. Corrales-Medina VF, Taljaard M, Yende S, et al. Intermediate and longterm risk of new-onset heart failure after hospitalization for pneumonia in elderly adults. Am Heart J. 2015;170(2):306-312.

4. Eurich DT, Marrie TJ, Minhas-Sandhu JK, Majumdar SR. Ten-year mortality after community-acquired pneumonia. A prospective cohort. Am J Respir Crit Care Med. 2015;192(5):597-604.

5. Eurich DT, Marrie TJ, Minhas-Sandhu JK, Majumdar SR. Risk of heart failure after community acquired pneumonia: prospective controlled study with 10 years of follow-up. BMJ. 2017;356:j413.

6. Restrepo MI, Faverio P, Anzueto A. Long-term prognosis in communityacquired pneumonia. Curr Opin Infect Dis. 2013;26(2):151-158.

7. Huang ST, Lin CL, Chang YJ, et al. Pneumococcal pneumonia infection is associated with end-stage renal disease in adult hospitalized patients. Kidney Int. 2014;86(5):1023-1030.

8. Hall MJ, DeFrances CJ, Williams SN, Golosinskiy A, Schwartzman A. National Hospital Discharge Survey: 2007 summary. Natl Health Stat Report. 2010;(29):1-20, 24.

9. Loeb M. Community-acquired pneumonia. BMJ Clin Evid. 2010;2010. pii: 1503 .

10. Gibson GJ, Loddenkemper R, Lundbäck B, Sibille Y. Respiratory health and disease in Europe: the new European Lung White Book. Eur Respir J. 2013;42(3):559-563.

11. O'Meara ES, White M, Siscovick DS, Lyles MF, Kuller LH. Hospitalization for pneumonia in the Cardiovascular Health Study: incidence, mortality, and influence on longer-term survival. J Am Geriatr Soc. 2005;53(7):1108-1116.

12. Kellum JA, Kong L, Fink MP, et al; GenIMS Investigators. Understanding the inflammatory cytokine response in pneumonia and sepsis: results of the Genetic and Inflammatory Markers of Sepsis (GenIMS) Study. Arch Intern Med. 2007;167(15):1655-1663.

13. Yende S, D'Angelo G, Kellum JA, et al; GenIMS Investigators. Inflammatory markers at hospital discharge predict subsequent mortality after pneumonia and sepsis. Am J Respir Crit Care Med. 2008;177(11):1242-1247.

14. Bash LD, Erlinger TP, Coresh J, Marsh-Manzi J, Folsom AR, Astor BC. Inflammation, hemostasis, and the risk of kidney function decline in the Atherosclerosis Risk in Communities (ARIC) Study. Am J Kidney Dis. 2009;53(4):596-605.

15. Shankar A, Sun L, Klein BE, et al. Markers of inflammation predict the long-term risk of developing chronic kidney disease: a population-based cohort study. Kidney Int. 2011;80(11):1231-1238.

16. Hiramoto JS, Katz R, Peralta CA, et al. Inflammation and coagulation markers and kidney function decline: the Multi-Ethnic Study of Atherosclerosis (MESA). Am J Kidney Dis. 2012;60(2):225-232.

17. Sundin PO, Udumyan R, Sjöström P, Montgomery S. Predictors in adolescence of ESRD in middle-aged men. Am J Kidney Dis. 2014;64(5): $723-729$. 
18. Jha V, Garcia-Garcia G, Iseki K, et al. Chronic kidney disease: global dimension and perspectives. Lancet. 2013;382(9888):260-272.

19. World Medical Association. World Medical Association Declaration of Helsinki: ethical principles for medical research involving human subjects. JAMA. 2013;310(20):2191-2194.

20. von Elm E, Altman DG, Egger M, et al; STROBE Initiative. The Strengthening the Reporting of Observational Studies in Epidemiology (STROBE) statement: guidelines for reporting observational studies. Ann Intern Med. 2007;147(8):573-577.

21. The National Patient Register [homepage on the Internet]. Stockholm: National Board of Health and Welfare. Available from: http://www. socialstyrelsen.se/register/halsodataregister/patientregistret/inenglish. Accessed May 2, 2018.

22. Ludvigsson JF, Andersson E, Ekbom A, et al. External review and validation of the Swedish national inpatient register. BMC Public Health. 2011;11:450.

23. Wettermark B, Hammar N, Fored CM, et al. The new Swedish Prescribed Drug Register--opportunities for pharmacoepidemiological research and experience from the first six months. Pharmacoepidemiol Drug Saf. 2007;16(7):726-735.

24. International Committee for Standardization in Haematology. Reference method for the erythrocyte sedimentation rate (ESR) test on human blood. Br J Haematol. 1973;24(5):671-673.

25. Whitworth JA; World Health Organization, International Society of Hypertension Writing Group. 2003 World Health Organization (WHO)/ International Society of Hypertension (ISH) statement on management of hypertension. J Hypertens. 2003;21(11):1983-1992.

26. Nordesjö L, Schéle R. Validity of an ergometer cycle test and measures of isometric muscle strength when prediction some aspects of military performance. Swed J Def Med. 1974;10:11-23.

27. Carlstedt B. Cognitive Abilities - Aspects of Structure, Process and Measurement. Göteborg: Göteborg studies in educational sciences, 0436-1121; 148, Acta Universitatis Gothoburgensis; 2000.

28. Student. The probable error of a mean. Biometrika. 1908;6(1):1-25.

29. Pearson K. X. On the criterion that a given system of deviations from the probable in the case of a correlated system of variables is such that it can be reasonably supposed to have arisen from random sampling. Lond Edinb Dubl Phil Mag. 1900;50(302):157-175.
30. Cox DR. Regression models and life-tables. $J$ R Stat Soc Series B Methodol. 1972;34(2):187-220.

31. Schoenfeld D. Partial residuals for The proportional hazards regression model. Biometrika. 1982;69(1):239-241.

32. May S, Hosmer DW. A simplified method of calculating an overall goodness-of-fit test for the Cox proportional hazards model. Lifetime Data Anal. 1998;4(2):109-120.

33. Wang MH, Shugart YY, Cole SR, Platz EA. A simulation study of control sampling methods for nested case-control studies of genetic and molecular biomarkers and prostate cancer progression. Cancer Epidemiol Biomarkers Prev. 2009;18(3):706-711.

34. Hennessy S, Bilker WB, Berlin JA, Strom BL. Factors influencing the optimal control-to-case ratio in matched case-control studies. Am J Epidemiol. 1999;149(2):195-197.

35. Xu H, Gasparini A, Ishigami J, et al. eGFR and the risk of communityacquired infections. Clin J Am Soc Nephrol. 2017;12(9):1399-1408.

36. McDonald HI, Thomas SL, Nitsch D. Chronic kidney disease as a risk factor for acute community-acquired infections in high-income countries: a systematic review. BMJ Open. 2014;4(4):e004100.

37. Cobo G, Hecking M, Port FK, et al. Sex and gender differences in chronic kidney disease: progression to end-stage renal disease and haemodialysis. Clin Sci (Lond). 2016;130(14):1147-1163.

38. Carrero JJ, Hecking M, Ulasi I, Sola L, Thomas B. Chronic kidney disease, gender, and access to care: a global perspective. Semin Nephrol. 2017;37(3):296-308.

39. Hwang SJ, Tsai JC, Chen HC. Epidemiology, impact and preventive care of chronic kidney disease in Taiwan. Nephrology (Carlton). 2010;15 Suppl 2:3-9.

40. Jha V, Prasad N. CKD and infectious diseases in Asia Pacific: challenges and opportunities. Am J Kidney Dis. 2016;68(1):148-160.

41. Arkwright PD, Abinun M, Cant AJ. Autoimmunity in human primary immunodeficiency diseases. Blood. 2002;99(8):2694-2702.

42. Halm EA, Fine MJ, Marrie TJ, et al. Time to clinical stability in patients hospitalized with community-acquired pneumonia: implications for practice guidelines. JAMA. 1998;279(18):1452-1457.

43. Hansson LO, Hedlund JU, Ortqvist AB. Sequential changes of inflammatory and nutritional markers in patients with community-acquired pneumonia. Scand J Clin Lab Invest. 1997;57(2):111-118.

\section{Clinical Epidemiology}

\section{Publish your work in this journal}

Clinical Epidemiology is an international, peer-reviewed, open access, online journal focusing on disease and drug epidemiology, identification of risk factors and screening procedures to develop optimal preventative initiatives and programs. Specific topics include: diagnosis, prognosis, treatment, screening, prevention, risk factor modification, systematic reviews, risk and safety of medical interventions, epidemiology and biostatistical methods, and evaluation of guidelines, translational medicine, health policies and economic evaluations. The manuscript management system is completely online and includes a very quick and fair peer-review system, which is all easy to use. 